consult they have exhausted their ability to cope with their child's illness independently. Parents need to know where, and when, to seek help and to be able to do so easily, without being judged for doing so. Services face the challenge of reducing barriers to timely treatment within financial constraints, if we are to reduce avoidable morbidity and mortality and improve UK child health outcomes.

\section{G28 EFFECTIVE SAFETY NETTING: AN IMPORTANT CONTRIBUTION TO AVOIDING PREVENTABLE DEATHS}

${ }^{1} \mathrm{SJ}$ Neill, ${ }^{2} \mathrm{CHD}$ Jones, ${ }^{3} \mathrm{D}$ Roland, ${ }^{4} \mathrm{M}$ Thompson, ${ }^{5} \mathrm{M}$ Lakhanpaul. ${ }^{1}$ School of Health, University of Northampton, Northampton, UK; ${ }^{2}$ Department of Primary Care Health Sciences, University of Oxford, Oxford, UK; ${ }^{3}$ Paediatric Emergency Medicine Leicester Academic Group, Department of Cardiovascular Sciences, Leicester University, Leicester, UK; ${ }^{4}$ Department of Family Medicine, University of Washington, Seattle, USA; ${ }^{5}$ General and Adolescent Paediatrics Unit, Institute of Child Health, University College London, London, UK

\subsection{6/archdischild-2015-308599.28}

Safety netting has conventionally been used during consultations when clinicians are uncertain about the trajectory of an illness to provide patients with guidance about when and where to re-consult (Roland et al 2013). Recent high profile events involving failure to recognise and appropriately safety net children with sepsis highlights this is a significant issue in healthcare.

Aim The aim of the paper is to raise awareness of the importance of quality safety netting information for parents of young children drawing on findings from the ASK SNIFF research programme.

Methods

1. Systematic integrative review of interventions designed to support families with an acutely sick child.

2. Qualitative study with 27 parents and 16 first contact health professionals using a grounded theory approach.

Results A review of over 7000 articles identified only 22 which examined an intervention educating and advising parents on when to seek medical care. Analysis of these papers showed that interventions which provide information on assessment and management and are endorsed by health care professionals (HCPs) have shown greatest effect. Irrelevant information appears to reduce parent's trust in the intervention. These findings validated the emerging themes from the qualitative work. Parents of sick children, in the latter, want information pre, during and post consultation with a HCP to inform their own safety netting decisions. Information is not routinely offered during consultations, is inconsistent in content and delivery method and often reported to be verbal only. The extent of safety netting was reported by HCPs to be influenced by parental status, an assumption of individual parent's knowledge and practical factors such as working printers. Both groups want safety netting tools to improve consistency and quality of information available for parents with a sick child.

Conclusion Safety netting information is essential for parents to know when to consult with a sick child. Absent or incomplete safety netting leaves parents to search elsewhere and with responsibility for assessing the reliability of information. Incomplete, inconsistent information may mislead and at best lead to increased consultations and at worst to delay and avoidable morbidity and mortality.

\section{G29}

AN EDUCATION PROGRAMME TO ENHANCE UNDERGRADUATE PRE-REGISTRATION NURSING STUDENTS' SKILLS IN RECOGNISING AND RESPONDING TO ACUTELY ILL/INJURED CHILDREN AND YOUNG PEOPLE: AN EVALUATION

SC Rogers, JM Hughes. School of Nursing, Midwifery and Social Work, University of Manchester, Manchester, UK

\subsection{6/archdischild-2015-308599.29}

Aim The aim of this paper is to report on the evaluation of an educational innovation designed to enhance pre-registration nursing students' knowledge and skills in recognising and responding to acutely ill/injured children and young people.

Background It is recognised that nurses are frequently one of the first note and observe the deterioration of a sick child and also may be the first to encounter a cardiac arrest (Nyman and Sihvonen 2000, Madden 2006). Literature indicates that student nurses require further education in the use and application of life support skills using supportive teaching strategies (NiemiMurola et al. 2007, Gordon and Buckley 2009); students in this cohort endorsed this in that they specifically identified these areas as vital for their future role as qualified nurses. A combination of simulation and problem based approaches has been found to be more effective than lecture based teaching alone (Wayne et al. 2005, Bruce et al. 2009, Szogedi et al. 2010).

Methods Final year pre-registration children's nursing students ( $\mathrm{n}=40$ ) on a BSc (Hons) Nursing programme completed an educational package delivered over four sessions. The training package consisted of a key note lecture, critical appraisal of clinical guidelines, seminar discussions of real life clinical scenarios, small group skills demonstrations and simulated teaching/testing using manikins. A five point Likert scale questionnaire, measuring students' self-perceived level of confidence, was used to evaluate the impact of the learning experience. Students completed a qualitative evaluation questionnaire as part of an end of unit evaluation.

Results Evaluation data revealed improvements in students' confidence, knowledge and skills. Comparisons of students' pre and post questionnaire scores revealed a statistically significant positive change in their self-perceived levels of confidence. The programme was positively evaluated as an educational package.

Conclusion This evaluation supports the role of a combination of teaching strategies to enhance knowledge and skills in undergraduate pre-registration nursing students in recognising and responding to acutely ill/injured children and young people.

\section{G30(P) ENHANCING THE PREPAREDNESS OF PARAMEDIC STUDENTS FOR MANAGING CHILDREN PRESENTING WITH CONGENITAL CARDIAC CONDITIONS IN THE PRE-HOSPITAL SETTING THROUGH AN ONLINE MULTIMEDIA EDUCATIONAL PACKAGE}

P Gregory. Institute of Health Professions, University of Wolverhampton, Wolverhampton, UK

\subsection{6/archdischild-2015-308599.30}

Paramedics are the front line clinicians in the delivery of prehospital emergency and urgent care in the UK and they face multiple challenges due to the unpredictability of their workload and the diversity of calls. Recently, proposed changes to children's cardiac services have prompted concerns that paramedics may attend an increasing number of children with congenital heart 